\title{
Transversity relations, chiral and holographic models, and pion wave functions from lattice QCD
}

\author{
Enrique Ruiz Arriola*† \\ Departamento de Física Atómica, Molecular y Nuclear, Universidad de Granada, \\ E-18071 Granada, Spain \\ E-mail: earriola@ugr.es
}

\section{Wojciech Broniowski}

The H. Niewodniczański Institute of Nuclear Physics, Polish Academy of Sciences, PL-31342 Kraków, Poland

Institute of Physics, Jan Kochanowski University, PL-25406 Kielce, Poland

E-mail: Wojciech.Broniowski@ifj.edu.pl

\begin{abstract}
We analyze the equal-time Bethe-Salpeter quark wave functions of the pion in various models. We discuss how the quenched lattice QCD results with delocalized pion interpolators can be identified with the coarse grained wave functions, typical of low-energy effective models. Actually, we find that one-loop chiral quark models predict that pseudoscalar and tensor wave functions have the same shape, while the axial component is more extended. These facts are accurately confirmed by the lattice. We also show how the transversity information, relevant for the light-cone physics, can be straightforwardly obtained from the equal-time rest-frame lattice calculations. This remarkable relation provides a way to extract, for instance, the equal-time holographic wave functions and compare them, quite favorably, to the lattice calculations.
\end{abstract}

Light Cone 2010 - LC2010

June 14-18, 2010

Valencia, Spain

\footnotetext{
*Speaker.

†Supported by the Spanish DGI and FEDER funds with grant FIS2008-01143/FIS, Junta de Andalucía grant FQM225-05, and EU Integrated Infrastructure Initiative Hadron Physics Project contract RII3-CT-2004-506078.

$¥$ Supported by Polish Ministry of Science and Higher Education, grants N N202 263438 and N N202 249235
} 


\section{Introduction}

Hadronic wave functions encode important information on bound states in strong-interaction physics; in particular, they provide the amplitude for a composite hadron to have quarks in a given momentum state or, equivalently, at a certain space-time distance. Heavy quarks obey nonrelativistic quantum mechanics and conserve particle number. While our understanding and intuition is based on wave functions, as a matter of principle the wave functions cannot be directly measured experimentally. One must instead resort to form factors, decay widths, or momentum distributions. Moreover, for light quark systems particle creation may occur, demanding a fieldtheoretic framework where further complications arise. Relativistic invariance requires that one uses the conventional Bethe-Salpeter (BS) amplitudes with a fixed number of the quark field operators, a reminiscent of the approximated parton picture point of view, emphasized by the light-cone approaches [1]. Color gauge invariance requires additional inclusion of the link operators [2].

For the pion, the spontaneously broken chiral symmetry is a basic dynamical ingredient in the determination of its nonperturbative quark structure. It appears via the pertinent axial WardTakahashi identities [3]. These important constraints are implemented in relativistic field-theoretic chiral quark models, such as the Nambu-Jona-Lasinio (NJL) model (for a review see, e.g., [4]). The regularization, introducing the physical cut-off, needs to be carefully handled not to spoil the relativistic, gauge, and chiral symmetries.

On the other hand, lattice QCD solves the bound state problem in a fundamental way. It is thus possible to make a first-principle nonperturbative determination of the wave functions, but at the expense of breaking the continuum symmetries, such as the Lorentz invariance and, quite often, chiral symmetry, due to the finite lattice spacing. The axial Ward-Takahashi identities can be exactly implemented on the discrete Euclidean lattice as shown by Ginsparg and Wilson [5] (see Ref. [6] for a recent practical implementation), enabling realistically small pion masses.

In the present contribution we show the analysis of the pion wave functions from the quenched lattice QCD [7] and make the comparison to various hadronic models. In spite of the very dissimilar appearance and nature of these approaches, we will provide the conditions under which this comparison may be undertaken. We also address in more detail the light-cone issues with the help of the transversity relations.

\section{Bethe-Salpeter Amplitudes}

The BS vertex or wave function of the pion is given by

$$
\chi_{p}(k)=-i \int d^{4} x e^{-i k \cdot x}\left\langle 0|T\{q(x) \bar{q}(0)\}| \pi_{a}(p)\right\rangle,
$$

where $q(x)$ are spinor field operators carrying flavor and color, and $\left|\pi_{a}(p)\right\rangle$ is the pion state with the Cartesian isospin index $a$ and the on-shell four-momentum $p, p^{2}=m_{\pi}^{2}$. While chiral quark model calculations are naturally formulated in the momentum space, the basic objects in Euclidean lattice calculations are the point-to-point correlation functions. These quantities are gauge and renormalization-group invariant at all Euclidean times, which basically correspond to off-shell processes. At large Euclidean times only the on-shell states contribute to the correlation functions, as 
well as the off-shell violations of the gauge invariance disappear. Inverting the Fourier transformation we get (in the isospin limit of $m_{u}=m_{d}$ )

$$
\left\langle 0|T\{q(x) \bar{q}(0)\}| \pi_{a}(q)\right\rangle=\frac{\tau_{a}}{f_{\pi}} \gamma_{5}\left[\Psi_{P}+\not \Psi_{A}+i \sigma^{\mu v} q_{\mu} x_{v} \Psi_{T}\right]
$$

where the wave functions $\Psi_{a}, a=P, A, T$, depend on the Lorentz-invariant variables $x^{2}, x \cdot q$, and $q^{2}=m_{\pi}^{2}$. The quantities $\Psi_{a}$ are vertex functions in the BS equation, and as such are finite and undergo $x$-independent multiplicative renormalization. Thus, the ratios $\Psi_{a}(x) / \Psi_{a}(0)$ become cutoff independent, as the cut-off is removed, which on the lattice means $a \rightarrow 0$.

The definition of Eq. (2.2) is completely satisfactory for chiral quark models. In QCD, however, it is only gauge-invariant in the fixed-point Fock-Schwinger gauge, $x^{\mu} A_{\mu}(x)=0$, where the standard derivatives, $\partial^{\mu}$, and the covariant derivatives, $D^{\mu}=\partial^{\mu}+i g A^{\mu}$, coincide. On the lattice the gauge fixing has the problem of the Gribov copies, as there is no complete gauge fixing. On the other hand, Elizur's theorem prevents non-vanishing vacuum expectation values of gauge variant operators in the physical Fock space.

Non-gauge invariant operators can be made gauge invariant by joining them with a link operator, however, as a result the path-dependence sets in. Furthermore, gluons carry momentum in the pion and different gauge-invariant definitions yield different results (see Ref. [8] for a discussion on various possibilities). For definiteness, we choose a straight-line path and undertake a smearing procedure. This delocalization improves the signal-to-noise ratio for the measured hadron correlators, as the interpolating operators have a larger overlap with the desired state. Local operators, in contrast, do not take into account the spatial extension of the hadron. The usefulness of the smearing process lies also in the fact that the overall thickness of the flux tube in the probe is controlled by the number of the smearing steps. In addition, the method is computationally simple. The resulting fat link also reduces the high-energy fluctuations and the path dependence, such that we deal with a coarse-grained wave function. The procedure naturally finds its counterpart in the low-energy effective chiral quark models. In a previous work [7] the quenched lattice calculations of the pion have been worked out along these lines. The quenched approximation contains all the leading- $N_{c}$, and hence the $\bar{q} q$, Fock state components. Thus we expect that quenched calculations describe the large- $N_{c}$ motivated models ${ }^{1}$.

\section{Transversity relations}

The relativistically invariant BS amplitude has the representation

$$
\begin{aligned}
\left\langle 0|T\{q(x) \bar{q}(0)\}| \pi_{a}(q)\right\rangle & =i \gamma_{5} \tau_{a} \int_{0}^{1} d \alpha e^{-i(2 \alpha-1) q \cdot x} \\
& \times\left[-\widetilde{\Psi}_{P}\left(\alpha, x^{2}\right)+\mathcal{q} \widetilde{\Psi}_{A}\left(\alpha, x^{2}\right)-2 i \sigma^{\mu v} q_{\mu} x_{v} \widetilde{\Psi}_{T}\left(\alpha, x^{2}\right)\right]
\end{aligned}
$$

where $\alpha$ is the Feynman parameter. As a matter of principle, all scalars such as $\Psi_{a}$ in Eq. (2.2) depend on the kinematic variables $x^{2}, x \cdot q$, and $q^{2}$, thus we are free to choose any form of kinematics.

\footnotetext{
${ }^{1}$ The quenched lattice calculations also contain a piece subleading in $N_{c}$, which is actually suppressed for heavy quarks; pion loops are $1 / N_{c}$-suppressed, although not all of the $1 / N_{c}$-contributions originate from the pion loops [9].
} 
In the rest-frame kinematics we have $x_{0}=0$ and $\left(q_{0}, \mathbf{q}\right)=\left(m_{\pi}, 0\right)$, whence $x^{2}=-\mathbf{x}^{2}, x \cdot q=0$, and $q^{2}=m_{\pi}^{2}$. On the other hand, in the infinite-momentum-frame kinematics $\left(q_{0}, \mathbf{q}\right)=\left(\sqrt{m_{\pi}^{2}+p_{z}^{2}}, p_{z}\right)$, with $p_{z} \rightarrow \infty$. Thus on the light-cone surface, $x_{0}=z$, one has $x^{2}=-\mathbf{x}_{T}^{2}$, and $x \cdot q \rightarrow 0$.

The Lorentz invariance allows us to relate the rest-frame calculation to the transverse-coordinate dependence in the light-cone wave functions. Simply, by comparing Eqs. (2.2) and (3.1) we find

$$
\Psi_{a}\left(x^{2}, x . q\right)=\int_{0}^{1} d \alpha \widetilde{\Psi}_{a}\left(\alpha, x^{2}\right) e^{-i q \cdot x(2 \alpha-1)} .
$$

We may identify the Feynman parameter $\alpha$ with the Bjorken $x$-variable, $x_{\mathrm{Bj}} \equiv \alpha$. Then, for the chosen kinematics $q^{+} x^{-}=q \cdot x=0$, we have $\Psi_{a}^{\mathrm{ET}}\left(-r^{2}, 0\right)=\int_{0}^{1} d x_{\mathrm{Bj}} \Psi_{a}^{\mathrm{LC}}\left(x_{\mathrm{Bj}},-r^{2}\right)$, where ET and $\mathrm{LC}$ denote the equal-time, and light-cone wave functions, respectively. In the argument of $\Psi^{\mathrm{ET}}$ one takes $r^{2}=\mathbf{x}^{2}$, the distance squared, while in the argument of $\Psi^{\mathrm{LC}}$ we need to use $r^{2}=\mathbf{x}_{T}^{2}$, the transverse distance squared. Therefore

$$
\Psi_{a}^{\mathrm{ET}}\left(-r^{2}, 0\right)=\left.\int_{0}^{1} d x_{\mathrm{Bj}} \Psi_{a}^{\mathrm{LC}}\left(x_{\mathrm{Bj}},-\mathbf{x}_{T}^{2}\right)\right|_{x_{T}=r} .
$$

That way the connection between the ET and LC wave functions has been established ${ }^{2}$.

Although generating the autonomous connection between LC and ET wave functions seems at first glance hopeless [10], a similar transversity relation has recently been deduced for scalar particles. Another transversity property for the Generalized Parton Distributions (GPDs) was suggested for the nucleon [11] and the pion [12], allowing a frame-independent definition of probability (unlike the more conventional Breit-frame definition). In the case of the pion it reads

$$
P^{\mathrm{LC}}(\mathbf{b})=\int_{0}^{1} d x q(x, \mathbf{b})=\int \frac{d^{2} q_{\perp}}{(2 \pi)^{2}} e^{i \mathbf{q}_{\perp} \cdot \mathbf{b}} F_{V}\left(-q_{\perp}^{2}\right)=\left.P^{\mathrm{ET}}(r)\right|_{r=b \equiv|\mathbf{b}|}
$$

where $q(x, \mathbf{b})$ is the off-forward diagonal GPD and $F_{V}(t)$ is the pion form factor. In the phenomenologically successful Vector Meson Dominance (VMD), where $F_{V}(t)=M_{V}^{2} /\left(M_{V}^{2}-t\right)$, one gets

$$
P^{\mathrm{ET}}(r)=M_{V}^{2} K_{0}\left(r M_{V}\right) /(2 \pi) \sim e^{-M_{V} r} / r^{\frac{1}{2}} .
$$

Besides these relations, it would also be useful to verify the ET-LC transversity connection directly on the lattice. While there exist transverse lattice calculations [13] (see [14] for a review), their focus is placed on the Distribution Amplitude, $\Psi(\alpha, 0)=\varphi(\alpha)$, leaving out the $x_{\perp}$ dependence.

\section{Chiral Quark Models vs Lattice}

We evaluate the correlation function of Eq. (2.2) in a chiral quark model (for a review see, e.g., [4]). Disregarding for the moment the regularization, an instructive way to determine the pion wave function in a chiral quark model is by exploiting the axial Ward-Takahashi identity. It relates the quark propagator, $S(p)$, and the vertex function corresponding to the axial current, $J_{A}^{\mu, a}(x)=\frac{1}{2} \bar{q}(x) \gamma^{\mu} \gamma_{5} \tau_{a} q(x)$, with the irreducible vertex $\Gamma_{A}^{\mu, a}(p+q, p)$ :

$$
S(p+q)^{-1} \gamma_{5} \frac{1}{2} \tau_{a}+\gamma_{5} \frac{1}{2} \tau_{a} S(p)^{-1}=q_{\mu} \Gamma_{A}^{\mu, a}(p+q, p) .
$$

\footnotetext{
${ }^{2}$ Note that we keep only the $\bar{q} q$ components of the BS amplitude, thus we do not account for the possible emergence of the higher Fock-state components, pertinent to the dynamical nature of the boost.
} 

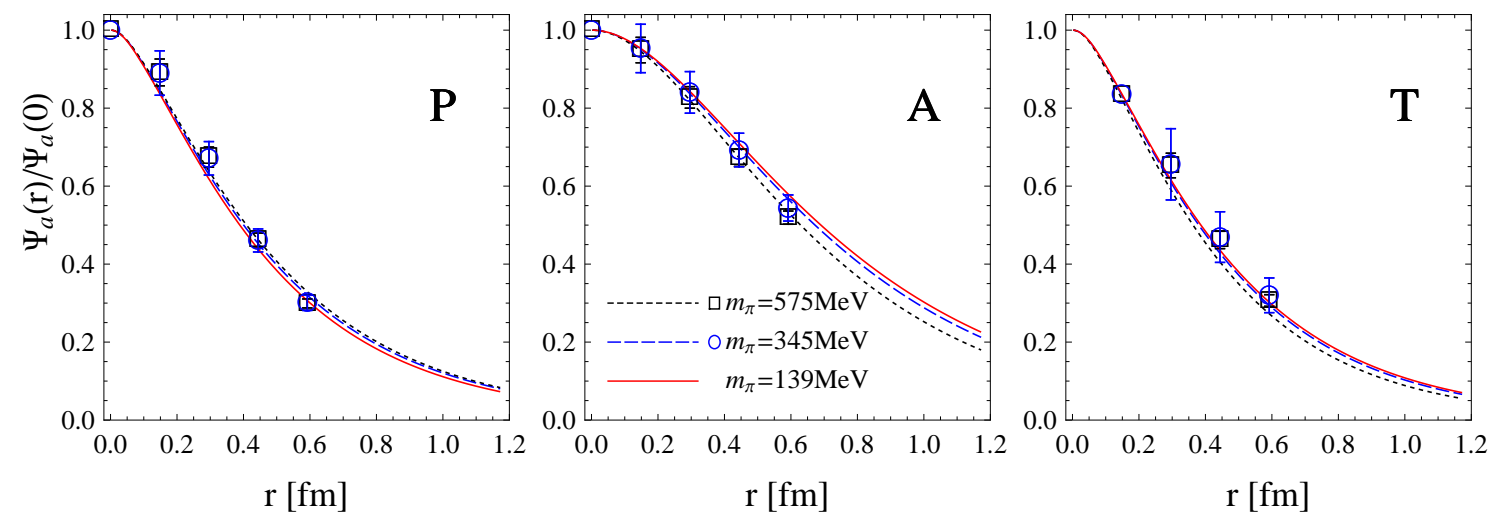

Figure 1: The components of the rest-frame equal-time pion wave function, normalized to unity at the origin, $\Psi_{a}(r) / \Psi_{a}(0)$, for $S, A$, and $T$ channels, evaluated in the NJL model at $M=g_{\pi q q} f_{\pi}=300 \mathrm{MeV}$, and compared to the quenched lattice data [7]. The displayed points of the lattice data are at $m_{\pi}=345$ and $575 \mathrm{MeV}$, while the NJL model calculation includes also the case of the physical pion mass.

In the NJL model, the spontaneous breaking of the chiral symmetry generates a constituent quark mass, $M$, given by the so-called gap equation. As the result, $S(p)=1 /(p-M)$, such that

$$
\Gamma_{A}^{\mu, a}(p+q, p)=\frac{\tau^{a}}{2} \gamma_{5}\left[\gamma^{\mu}-\frac{q^{\mu}}{q^{2}} \frac{2 M}{f_{\pi}}\right]
$$

The pole at $q^{2}=0$ indicates the Goldstone boson nature of the pion. The pion wave function is extracted from the pion pole as an unamputated vertex function,

$$
\chi_{q}^{a}(k)=\frac{i}{k+q-M}\left(\frac{M}{f_{\pi}} \gamma_{5} \tau_{a}\right) \frac{i}{k-M},
$$

where the Goldberger-Treiman relation at the quark level, $g_{\pi q q}=M / f_{\pi}$, can be read off. With the Feynman trick, the result becomes particularly simple in the chiral limit $q^{2}=m_{\pi}^{2} \rightarrow 0$, yielding

$$
\begin{aligned}
& \widetilde{\Psi}_{P}\left(\alpha, x^{2}\right)=M\left[-2(2 \alpha-1) \partial_{x^{2}}+\partial^{\mu} \partial_{\mu}+M^{2}\right] \partial_{M^{2}} \Delta(M, x), \\
& \widetilde{\Psi}_{A}\left(\alpha, x^{2}\right)=M^{2} \partial_{M^{2}} \Delta(M, x), \\
& \widetilde{\Psi}_{T}\left(\alpha, x^{2}\right)=-2 \partial_{x^{2}} \partial_{M^{2}} \Delta(M, x),
\end{aligned}
$$

where we have introduced the free scalar propagator in the coordinate space,

$$
\Delta(M, x)=\int \frac{d^{4} p}{(2 \pi)^{4}} \frac{e^{i p \cdot x}}{p^{2}-M^{2}}=\frac{M K_{1}\left(M \sqrt{-x^{2}}\right)}{4 \pi^{2} \sqrt{-x^{2}}} .
$$

From the previous formulas we find (for $m_{\pi}=0$ ) the relations

$$
\begin{array}{r}
\Psi_{P}(r)=2 \Psi_{T}(r)=\left.\frac{g_{\pi q q} N_{c} M}{\pi^{2} r} K_{1}(M r)\right|_{\mathrm{reg}} \sim \frac{e^{-M r}}{r^{3 / 2}}, \\
\Psi_{A}(r)=\left.\frac{g_{\pi q q} M N_{c}}{2 \pi^{2}} K_{0}(M r)\right|_{\mathrm{reg}} \sim \frac{e^{-M r}}{r^{1 / 2}},
\end{array}
$$

where $K_{0}$ and $K_{1}$ are the modified Bessel functions and "reg" means a regulator. The asymptotic behavior at $r \rightarrow \infty$ is independent of the regulator and implies a longer tail in the $A$ channel than 


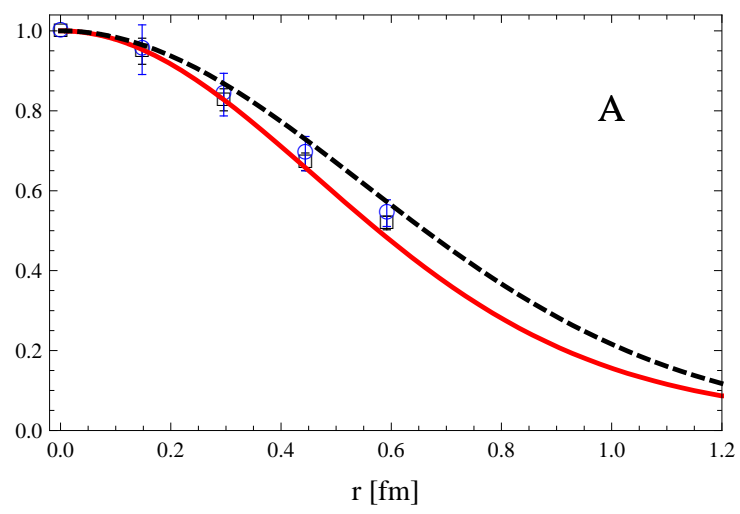

Figure 2: Axial equal-time holographic wave functions, normalized to unity at the origin, for the quark masses $m=330 \mathrm{MeV}$ (dashed black) and $m=4 \mathrm{MeV}$ (solid red), obtained via the transversity relations and compared to the lattice calculation with $m_{\pi}=575 \mathrm{MeV}$ (boxes) and $345 \mathrm{MeV}$ (circles) [7].

in the $P$ and $T$ channels. The exponential fall-off of the pion wave functions is consistent, up to a power in $\mathrm{r}$, with the probabilistic estimate in Eq. (3.5) with $M_{V}=2 M, M$ being the constituent mass. At short distances as $\Delta\left(x^{2}, M^{2}\right) \sim 1 / x^{2}$ the results are divergent demanding regularization.

The NJL model with the (twice subtracted) Pauli-Villars (PV) regularization applied to an observable $A$ amounts to the replacement $M^{2} \rightarrow M^{2}+\Lambda^{2}$, followed by the subtraction

$$
\left.A\right|_{\mathrm{reg}}=A\left(\Lambda^{2}=0\right)-A\left(\Lambda^{2}\right)+\Lambda^{2} \frac{d A\left(\Lambda^{2}\right)}{d \Lambda^{2}} .
$$

Results of the calculation, shown already in [7], are given in Fig. 1. As we see, the agreement is excellent. At the farthest lattice point, $r=0.6 \mathrm{fm}$, the ratio $r \Psi_{A}(r) / \Psi_{P}(r)$ approaches a constant.

The quantitative agreement of the NJL model with the data is not trivial. For instance, in the Spectral Quark Model (SQM) [15] only qualitative matching is achieved. In this approach the regularization is introduced by replacing the mass $M$ by the "spectral" mass $\omega$, using $g_{\pi q q}=\omega / f_{\pi}$ and integrating over $\omega$ with a suitable weight $\rho(\omega)$, which depends on the vector and scalar meson masses $\left(M_{V}\right.$ and $\left.M_{S}\right)$, and also on a specified contour in the complex $\omega$-plane. That way, for instance, VMD of the pion form factor can be built in with $M_{V}^{2}=24 \pi^{2} f_{\pi}^{2} / N_{c}$. For $m_{\pi}=0$ we get the results

$$
\frac{\Psi_{P}(r)}{\Psi_{P}(0)}=\frac{\Psi_{T}(r)}{\Psi_{T}(0)}=e^{-M_{S} r / 2}, \quad \frac{\Psi_{A}(r)}{\Psi_{A}(0)}=e^{-M_{V} r / 2}\left(1+\frac{M_{V} r}{2}\right) .
$$

Again, $\Psi_{A}$ is more extended than $\Psi_{P}$ and $\Psi_{T}$, due to the presence of an extra power in $r$. In the case of SQM we would get good fits of the lattice data for $M_{V} / 2=505(30), 520(20), 530(14) \mathrm{MeV}$ for the subsequent values of $m_{\pi}=345,475,575 \mathrm{MeV}$. A simple quadratic extrapolation in $m_{\pi}$ to the chiral limit yields $M_{V} / 2=493(20) \mathrm{MeV}$, a too high value as compared to $M_{V}=m_{\rho}=770 \mathrm{MeV}$.

\section{Holographic wave functions vs lattice}

The transversity relations (3.3) can be used to deduce the ET wave functions from the LC wave functions. As we have shown, ET wave functions can be computed on Euclidean lattices upon a suitable coarse graining of the gauge link operator. As an example, we take here the holographic 
wave functions inspired by the AdS/CFT correspondence and recently brought in connection with the LC wave functions [16] as a first approximation to QCD (for a review see e.g. [17]). The basic idea was to relate the QCD-LC Hamiltonian by using the scaling relation between the twodimensional vectors $\zeta=\sqrt{x(1-x)} \mathbf{b}$ (valid for massless quarks) and assume that the interaction also depends on this scaling variable. Further elaborated models with finite quark masses are introduced, assuming the replacement $\mathbf{k}_{\perp}^{2} \rightarrow \mathbf{k}_{\perp}^{2}+m^{2}$ [18]. However, by doing so it is not obvious whether $m$ corresponds to the current or constituent mass of the quark. For that reason, masses $m=$ $4 \mathrm{MeV}$ and $m=330 \mathrm{MeV}$ are explored. A full discussion of models is carried out in [19], where the soft-wall with a positive dilaton background seems phenomenologically preferred. Actually, besides the good quality of the mass spectrum, $M_{n L S}^{2}=2 \pi \sigma(n+L+S / 2)$ with $4 \kappa^{2}=2 \pi \sigma$, the pion arises as a massless mode (corresponding to $n=L=S=0$ ). Unlike the chiral models, this is not linked to the spontaneous breaking of the chiral symmetry, apparently not manifest in the light-cone dynamics. Thus, we identify the BS axial component with the holographic wave function $[16,18]$

$$
\Psi_{A}^{\mathrm{LC}}\left(\mathbf{b}_{\perp}, x\right)=\frac{A \kappa}{\sqrt{\pi}} \sqrt{x(1-x)} \exp \left[-\frac{\kappa^{2}}{2} x(1-x) \mathbf{b}_{\perp}^{2}-\frac{m^{2}}{2 \kappa^{2} x(1-x)}\right] .
$$

Calculations can be undertaken analytically for massless quarks. Following [18] we fix the pion weak decay, $\pi^{+} \rightarrow \mu^{+} \bar{v}$, and the neutral pion decay, $\pi^{0} \rightarrow 2 \gamma$, from the conditions

$$
\left.\frac{1}{2 \sqrt{\pi}} \int_{0}^{1} d x \Psi_{A}^{\mathrm{LC}}\left(\mathbf{b}_{\perp}, x\right)\right|_{\mathbf{b}_{\perp}=0_{\perp}}=\frac{f_{\pi}}{2 \sqrt{3}}=\frac{A \kappa}{16}, \quad 2 \sqrt{\pi} \int_{0}^{1} d x d^{2} \mathbf{b}_{\perp} \Psi_{A}^{\mathrm{LC}}\left(\mathbf{b}_{\perp}, x\right)=\frac{\sqrt{3}}{f_{\pi}}=\frac{4 A \pi^{2}}{\kappa}
$$

respectively, with $f_{\pi}=92.4 \mathrm{MeV}$. This yields $A^{2}=2 / \pi^{2}=0.2$ and $\kappa=4 \pi \sqrt{2 / 3} f_{\pi}=950 \mathrm{MeV}$. Using the transversity relation the ET (holographic) wave function reads (in the massless case, $m=0$ )

$$
\frac{\Psi_{A}^{\mathrm{ET}}(r)}{\Psi_{A}^{\mathrm{ET}}(0)}=e^{-2 f_{\pi}^{2} \pi^{2} r^{2} / 3}\left[I_{0}\left(2 f_{\pi}^{2} \pi^{2} r^{2} / 3\right)-I_{1}\left(2 f_{\pi}^{2} \pi^{2} r^{2} / 3\right)\right]=1-\pi^{2} f_{\pi}^{2} r^{2}+\mathscr{O}\left(r^{4}\right),
$$

where $I_{n}(z)$ are the modified Bessel functions. Note that upon the use of the relation $M_{V}^{2}=$ $24 \pi^{2} f_{\pi}^{2} / N_{c}$ the small-r expansion reproduces the SQM result, Eq. (4.8), exactly. The asymptotic behavior at large distances has the form

$$
\frac{\Psi_{A}^{\mathrm{ET}}(r)}{\Psi_{A}^{\mathrm{ET}}(0)}=\frac{3 \sqrt{3}}{8 f^{3} \pi^{7 / 2}}\left(\frac{m}{r^{2}}+\frac{1}{r^{3}}+\mathscr{O}\left(r^{-5}\right)\right) e^{-m r},
$$

which displays the exponential fall-off, similarly to the chiral quark models, Eq. (4.6) for NJL and Eq. (4.8) for SQM, however the powers of $r$ are different, exhibiting different dynamics in the models. The parameters $A$ and $\kappa$ for $m=4 \mathrm{MeV}$ and $m=330 \mathrm{MeV}$ in Eq. (5.1) are taken as in [18]). As we can see from Fig. 2, the agreement is quite good and the lattice data hardly allow to discriminate between the mass values except at $r=0.6 \mathrm{fm}$. Of course, it would be very useful to pin-down the correct long distance behavior from the lattice above $0.6 \mathrm{fm}$. The calculation of the other (higher-twist) components within the holographic approach would also be highly desirable.

Our numerical study shows that the lattice data in Fig. 2 can be best fitted with $m \sim 100 \mathrm{MeV}$, similar to the current quark mass used in the NJL calculation at high values of $m_{\pi}$. In particular, for $m_{\pi}=575$ and $345 \mathrm{MeV}$ we have used $m=140$ and $51 \mathrm{MeV}$, respectively, in rough agreement with the Gell-Mann-Oakes-Renner relation $-m\langle\bar{q} q\rangle=f_{\pi}^{2} m_{\pi}^{2}$. Thus, the interpretation of $m$ in the holographic models as the current quark mass seems consistent with this comparison. 


\section{Conclusions}

The presented calculations show that the Euclidean lattices can be successfully used to coarse grain the wave function over short-distance scales, where the gluon degrees of freedom are integrated out. A direct comparison to wave functions from various hadronic models not only becomes meaningful, but in some cases very successful. A quite unexpected result concerns the utility of our calculations to determine the transversity information (the dependence on the transverse coordinates), relevant for the light-cone physics; the infinite-momentum frame pion wave functions, integrated over the Bjorken- $x$, coincide in the impact-parameter space with the equal-time restframe wave functions. This relation provides a way of checking the wave functions for models genuinely formulated in the LC variables. As an example, we have carried out this analysis for holographic models.

We thank Sasa Prelovsek and Luka Santelj for their collaboration in the lattice calculation [7].

\section{References}

[1] S. J. Brodsky, H.-C. Pauli and S. S. Pinsky Phys. Rept. 301 (1998) 299-486 [hep-ph/ 9705477$].$

[2] H. Suura Phys. Rev. D17 (1978) 469.

[3] H. Pagels and S. Stokar Phys. Rev. D20 (1979) 2947.

[4] E. Ruiz Arriola Acta Phys. Polon. $B 33$ (2002) 4443-4479 [hep-ph/ 0210007$].$

[5] P. H. Ginsparg and K. G. Wilson Phys. Rev. D25 (1982) 2649.

[6] C. Gattringer Phys. Rev. D63 (2001) 114501 [hep-lat/0003005].

[7] W. Broniowski, S. Prelovsek, L. Santelj and E. R. Arriola Phys. Lett. B686 (2010) 313-318 [0911.4705].

[8] J. W. Negele hep-lat/0007026.

[9] T. D. Cohen and D. B. Leinweber Comments Nucl. Part. Phys. 21 (1993) 137-150 [hep-ph/9212225].

[10] G. A. Miller and B. C. Tiburzi Phys. Rev. C81 (2010) 035201 [0911. 3691].

[11] G. A. Miller Phys. Rev. Lett. 99 (2007) 112001 [0705.2409].

[12] W. Broniowski, E. R. Arriola and K. Golec-Biernat Phys. Rev. D77 (2008) 034023 [0 712 . 1012].

[13] S. Dalley and B. van de Sande Phys. Rev. D67 (2003) 114507 [hep-ph/ 0212086$].$

[14] M. Burkardt and S. Dalley Prog. Part. Nucl. Phys. 48 (2002) 317-362 [hep-ph/ 0112007 ].

[15] E. Ruiz Arriola and W. Broniowski Phys. Rev. D67 (2003) 074021 [hep-ph/0301202].

[16] G. F. de Teramond and S. J. Brodsky Phys. Rev. Lett. 102 (2009) 081601 [080 9 . 4899].

[17] S. J. Brodsky and G. F. de Teramond 1009.4232.

[18] A. Vega, I. Schmidt, T. Branz, T. Gutsche and V. E. Lyubovitskij Phys. Rev. D80 (2009) 055014 [0906.1220].

[19] S. S. Afonin 1001.3105. 